Verbeek-van Noord, I., Bruijne, M.C. de, Twisk, J.W.R., Dyck, C. van, Wagner, C. More explicit communication after classroom-based crew resource management training: results of a pragmatic trial. Journal of Evaluation in Clinical Practice: 2015, 21(1), 137-144

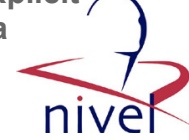

\begin{tabular}{|l|l|}
\hline $\begin{array}{l}\text { Postprint } \\
\text { Version }\end{array}$ & 1.0 \\
\hline Journal website & $\frac{\text { http://onlinelibrary.wiley.com/doi/10.1111/jep.12261/abstract;jsessionid=49D802 }}{\text { E6206227819C00946E646D910B.f03t04 }}$ \\
\hline Pubmed link & $\underline{\text { http://www.ncbi.nlm.nih.gov/pubmed/25314899 }}$ \\
\hline DOI & $10.1111 /$ jep.12261
\end{tabular}

This is a NIVEL certified Post Print, more info at http://www.nivel.eu

\title{
More explicit communication after classroom- based crew resource management training: results of a pragmatic trial
}

InGE VERBEEK-VAN NOORD PHD POST-DOCTORAL RESEARCHER ${ }^{1, *}$, MARTINE C. DE BRUiJne MD, PHD AsSOCIATE Professor ${ }^{1}$, JOS W. R. TWISK PHD PROFESSOR OF APPLIED BIOSTATISTICS ${ }^{2}$, CATHY VAN DYCK PHD ASSOCIATE PROFESSOR ${ }^{3}$ AND CORDULA WAGNER PHD PROFESSOR OF PATIENT SAFETY ${ }^{1,4}$

1 Department of Public and Occupational Health, EMGO Institute for Health and Care Research, VU University Medical Center, Amsterdam, The Netherlands

2 Department of Epidemiology and Biostatistics, EMGO Institute for Health and Care Research, VU University Medical Center, Amsterdam, The Netherlands

3 Department of Organization Sciences, VU University Amsterdam, Amsterdam, The Netherlands

4 NIVEL Netherlands Institute for Health Services Research, Utrecht, The Netherlands

\begin{abstract}
Rationale, aims and objectives: Aviation-based crew resource management trainings to optimize non-technical skills among professionals are often suggested for health care as a way to increase patient safety. Our aim was to evaluate the effect of a 2-day classroom-based crew resource management (CRM) training at emergency departments (EDs) on explicit professional oral communication (EPOC; non-technical skills).

Method: A pragmatic controlled before-after trial was conducted. Four EDs of general teaching hospitals were recruited (two intervention and two control departments). ED nurses and ED doctors were observed on their non-technical skills by means of a validated observation tool (EPOC). Our main outcome measure was the amount of EPOC observed per interaction in 30 minutes direct observations. Three outcome measures from EPOC were analysed: human interaction, anticipation on environment and an overall EPOC score. Linear and logistic mixed model analyses were performed. Models were corrected for the outcome measurement at baseline, days between training and observation, patient safety culture and error management culture at baseline.

Results: A statistically significant increase after the training was found on human interaction $(\beta=0.27,95 \%$ CI $0.08-0.49)$ and the overall EPOC score $(\beta=0.25,95 \%$ CI $0.06-0.43)$, but not for anticipation on environment
\end{abstract}


Verbeek-van Noord, I., Bruijne, M.C. de, Twisk, J.W.R., Dyck, C. van, Wagner, C. More explicit communication after classroom-based crew resource management training: results of a pragmatic trial. Journal of Evaluation in Clinical Practice: 2015, 21(1), 137-144

$(\mathrm{OR}=1.19,95 \%$ CI $.45-3.15)$. This means that approximately $25 \%$ more explicit communication was shown after CRM training.

Conclusions: We found an increase in the use of CRM skills after classroombased crew resource management training. This study adds to the body of evidence that CRM trainings have the potential to increase patient safety by reducing communication flaws, which play an important role in health carerelated adverse events.

\section{INTRODUCTION}

Since the Institute of Medicine encouraged hospitals to become High Reliability Organizations [1], crew resource management (CRM) training has been recommended for use in health care [2,3]. CRM training is an educational training intended to change trainees' attitudes towards safety principles and to stress the importance of non-technical skills. This should lead to increased team performance, better decision making and higher safety levels.

CRM training in health care includes classroom-based or simulation-based training, or a combination of both. These courses are predominantly given to units at risk for human error with severe consequences, such as emergency departments (EDs) [4] because of, for example, high cognitive workload, high levels of uncertainty, and many interruptions and distractions [5].

The working mechanism of CRM training centres on situation awareness (SA), which is defined as 'the perception of the elements in the environment within a volume of time and space, the comprehension of their meaning, and the projection of their status in the near future' [6]. Maintaining an accurate SA is a key challenge for optimal decision making in highly dynamic situations [7]. To achieve this level of accuracy, input from the environment is required, particularly by means of more explicit communication among team members.

Prior studies have shown that CRM training may have positive effects on participants' attitudes and behaviour [8]. Two studies assessed the effect on behaviour in a controlled study and showed positive results $[9,10]$. Therefore, we conducted an evaluation study for the ED in the Dutch health care setting. During this study, we adjusted for specific safety culture dimensions since safety culture influences the effectiveness of CRM implementation [11]. For example, removing disincentives for speaking up relates to the successful implementation of CRM [12]. The specific aim of the current study was to evaluate whether non-technical skills are more often observed after a classroom-based CRM training to all ED caregivers, when controlled for safety culture dimensions prior to the intervention.

\section{METHODS AND MATERIALS}

The medical ethical committee of the VU University Medical Center and the Scientific Committee of the EMGO Institute for Health and Care Research approved the study (reference number 2007-028) An exemption of the medical ethical committee of the VU University Medical Center was obtained, which stated that, according to the Dutch Medical Research and Human Experimentation Law, no formal declaration was necessary since no patients were involved. 
Verbeek-van Noord, I., Bruijne, M.C. de, Twisk, J.W.R., Dyck, C. van, Wagner, C. More explicit communication after classroom-based crew resource management training: results of a pragmatic trial. Journal of Evaluation in Clinical Practice: 2015, 21(1), 137-144

\section{Study design}

A pragmatic controlled before-after trial was conducted with two EDs in the intervention group and two in the control group.

\section{Setting}

In the Netherlands, it has been recognized that emergency medicine is an important discipline in itself, illustrated by the founding of the Dutch College of Emergency Physicians in 1999 and the Society for Training in Emergency Medicine in 2004. At present, many EDs are working with specialized ED doctors whose training is based on the residency curriculum of the American Board of Emergency Medicine. Care that has to be provided in the ED is generally structured similarly among all Dutch EDs. Patient are first triaged. Next, a coordinating nurse allocates patients to ED rooms. Then, nurses see the patients and first tests are done according to local protocols, after which doctors see the patients. Care in the ED is, thus, very sequenced and as a result care is characterized by a lack of communication between the health care professionals. Handover occurs more often by means of written notes than face to face. Briefing of all involved professionals occurs only when patients need acute complex care delivered by a team, such as in the case of high-energy traumas.

The EDs in our study were based in non-academic, general Dutch teaching hospitals that all employed emergency doctors. Patient censuses were between 20000 and 30000 visits. Co-interventions focusing on non-technical skills were avoided during the study period. Furthermore, EDs were expected not to have reorganizations, such as management switches.

\section{Selection of emergency departments and trainees}

With the help of the Dutch Association of Medical Specialists (DAMS), four EDs were recruited. Three EDs enrolled in response to a letter of the DAMS. One ED enrolled after an informal conversation between a researcher and the head of the department. Allocation to either the intervention or control group was based on readiness to schedule staff for a 2-day CRM training course during working hours. EDs in the control group were offered the training against the same fee as the departments in the intervention group after the study period.

All staff with over 6 months of work experience and being likely to still be working in the ED during the post-training measurement period were included in the study. Participants were being informed by means of an information folder and an invitational oral presentation. Participation in the training was mandatory. One trainer did pre-training interviews and on-site observations at intervention EDs to assess specific needs. Training took place in multi-professional groups of 9 to 16 persons. At one intervention site, doctors from anaesthesia and surgery joined. All professionals from one intervention department were trained $(n=39)$. At the other intervention department, 31 professionals were trained and 18 professionals were not trained, given the above-mentioned inclusion criteria. In total, 70 persons were allocated to seven training groups.

\section{Intervention}


Verbeek-van Noord, I., Bruijne, M.C. de, Twisk, J.W.R., Dyck, C. van, Wagner, C. More explicit communication after classroom-based crew resource management training: results of a pragmatic trial. Journal of Evaluation in Clinical Practice: 2015, 21(1), 137-144

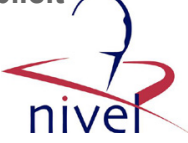

The intervention comprised a 2-day classroom-based course given by two trainers from a commercial vendor with experience in training aviation personnel as well as health care personnel. The training used adult learning principles in which participants were welcomed to bring in their experience and to reflect on individual and group processes in a safe environment. During the training, the trainers had the opportunity to skip a few topics or to go deeper into another, but in general training groups received the same training (Table 1). Educational tools included lectures, facilitated discussions, video clips, assignments and exercises. The goal of the course was to increase participants' awareness of how errors develop and how they could be managed through the use of team competencies. Furthermore, at the level of the individual, the team and the organization, projects for improvement were specified. Training was given between the end of January 2009 and the beginning of April 2009. EDs had the opportunity to schedule two voluntary post-training meetings 4 weeks after the training, coached by the CRM trainer to discuss problems implementing CRM principles and projects for improvement.

\section{[TABLE 1]}

\section{Direct observations}

Using a preliminary version of the explicit professional oral communication (EPOC) observation form (EPOC) [13], health care workers were directly observed by independent observers $(n=2)$ with a background in health sciences or organizational psychology. Six key dimensions were observed: assertiveness, working with others, people-oriented leadership, task-oriented leadership, situation awareness, and planning and anticipating. Those six dimensions could be subdivided into three categories, self, human interaction (HI) and anticipation on environment (AoE; Table 3).

Each time a health care worker showed a specific item of EPOC, this was scored. Furthermore, EPOC kept track of the number of work-related interactions during the observation period. Interobserver reliability appeared high with an intraclass correlation (ICC) of 0.91 (95\% CI 0.84-0.95) for EPOC overall, 0.90 (95\% CI 0.80$0.95)$ for HI and 0.85 (95\% CI 0.75-0.91) for AoE [13]. These ICCs were calculated on the same data that was used in this study.

We aimed to observe health care workers between one and three times during both measurement periods. Observations lasted half an hour each, with two observers independently observing ED personnel during their work in the ED. Observers scheduled the observations based on the presence of nurses and doctors on the particular observation day between 1100 and 1800 hours. ED personnel could refuse observation at any time.

\section{Outcome measures}

Use of non-technical skills was assessed by the average number of tallies (number of times items of EPOC were seen) observed per interaction within a 30 minutes observation of an individual staff member. Our outcome parameters were an overall sum score of all EPOC items together and sum scores on the primary dimensions of EPOC: self, HI and AoE. 
Verbeek-van Noord, I., Bruijne, M.C. de, Twisk, J.W.R., Dyck, C. van, Wagner, C. More explicit communication after classroom-based crew resource management training: results of a pragmatic trial. Journal of Evaluation in Clinical Practice: 2015, 21(1), 137-144

\section{Safety culture assessment}

The validated Dutch version [14] of the Hospital Survey on Patient Safety Culture (HSOPSC) [15] was used to measure patient safety culture. This questionnaire measures 11 patient safety culture dimensions. We selected relevant patient safety culture dimensions on a previous study [16], which showed that smooth transitions, frequency of event reporting, non-punitive response to error, feedback about and learning from error, hospital management support and adequate staffing were preconditions for implementing patient safety improvement strategies. Additionally, the Error Culture Questionnaire (ECQ) was used to assess error management culture, which is an extension and adaptation $[17,18]$ of the Error Orientation Questionnaire [19] and consists of 47 items covering four dimensions, which in turn cover two or three sub-dimensions each. 'Mastery' is formed by analysing, learning from and correcting errors. 'Social orientation' consists of communication about errors and helping each other after an error occurred. 'Awareness' addresses anticipation on errors, acceptance of errors and risk taking. 'Aversion' covers rigidity in preventing errors, strain after an error occurred and covering up after an error occurred.

\section{Data collection and processing}

Pre- and post-training data were collected between November 2008 and January 2009, and between May and August 2009, respectively. Measurements were done contemporaneously in one intervention and one control group and lasted 2 months each.

\section{Data analysis}

The effect of the intervention was analysed with mixed model analyses using MLwiN version 2.22 (Centre for Multilevel Modelling, University of Bristol, Bristol, UK). Mixed models were used because observations were clustered within subjects. For the EPOC overall score and HI, linear mixed model analyses were performed. As scores on AoE were not normally distributed, logistic mixed model analyses were performed for AoE.

Four models were built stepwise: (1) a crude model adjusted for the baseline measurement of the particular outcome variable; (2) additionally adjusted for the days between training and observation; (3) additionally adjusted for relevant patient safety culture dimensions from the HSOPSC at baseline; and (4) additionally adjusted for the error management culture dimensions from the ECQ at baseline. To assess the influence of missing data, we compared results based on all available observations without correction for culture measurements with those of the participants who completed all measurements on both culture questionnaires. At baseline, 149 persons (126 nurses and 23 emergency doctors) were included as subjects that could be observed (79 in the intervention group and 70 in the control group), excluding managers and secretaries. From those 149 subjects, 10 (6.7\%) were not observed during post-training measurement as they had left the organization. Additionally, $10(6.7 \%)$ were not observed during baseline measurement, $15(10.0 \%)$ were not observed during post-training measurement, and $29(19.5 \%)$ were not observed at all as they were not present at the right time. Only occasionally, a caregiver refused to participate. Table 2 gives an overview of the 
Verbeek-van Noord, I., Bruijne, M.C. de, Twisk, J.W.R., Dyck, C. van, Wagner, C. More explicit communication after classroom-based crew resource management training: results of a pragmatic trial. Journal of Evaluation in Clinical Practice: 2015, 21(1), 137-144

number of persons that were observed and the number of observations and the use of the corresponding data in the analyses. No significant differences were found between respondents that were observed both measurement periods and those with complete data on safety culture regarding their profession and their membership to the intervention or control group.

\section{[TABLE 2][TABLE 3]}

After the intervention, 86 persons were observed in total, $82(95.3 \%)$ nurses and four ED doctors (4.7\%), of which 65 completed both culture surveys at baseline. This left us with $62(95.4 \%)$ nurses and $3(4.6 \%)$ doctors for the fully adjusted analyses. On average, observed persons in the intervention groups saw 2.0 patients per half an hour at baseline and 2.1 in the post-intervention period. These numbers were 2.1 and 2.0 for the control group, respectively. No observable differences were found in triage classification of patients. Most patients fell in the yellow (must see within 1 hour) and green (must see within 2 hours) category in both groups during both measurement periods.

Table 3 demonstrates the number of interactions between the observed person and other health care workers and the amount of explicit professional communication in both groups. The average number of interactions per half an hour observation before and after intervention differed between groups, but not within groups over time. Items belonging to the assertiveness dimensions were hardly seen. The dimensions of the HI category were predominantly observed.

Table 4 displays the training effect based on all available observation data adjusted for the particular outcome variable at baseline and additionally for the days between training and observation, but not adjusted for organizational culture. The EPOC category self was hardly observed and therefore excluded from the analyses.

Observations were not clustered within individuals. A significant intervention effect was found for the EPOC overall score and the HI subset score, but not for the AoE subset score. Correction for the days between the training and the observation had a confounding effect as the intervention effect increased from 0.14 (95\% CI $0.02-0.26)$ to 0.19 (95\% CI $0.07-0.31)$. Furthermore, the days between training and observations had a positive effect on both the EPOC overall score $(\beta=0.005,95 \% \mathrm{CI}$ 0.001-0.009) and HI subset score $(\beta=0.005,95 \%$ CI $0.001-0.009)$, but no significant interaction with training was seen. Thus, with an increase in the number of days after the intervention, more explicit communication was seen.

\section{[TABLE 4]}

Table 5 reports the culture covariates of those respondents who were observed during both measurement periods and who filled in both culture questionnaires. At baseline, significant differences between intervention and control group existed.

\section{[TABLE 5]}

Table 6 shows the results of the intervention effect adjusted for the potential confounders described in Table 5. Adjustment for patient safety and error management culture dimensions led to an increase of the intervention effect 
Verbeek-van Noord, I., Bruijne, M.C. de, Twisk, J.W.R., Dyck, C. van, Wagner, C. More explicit communication after classroom-based crew resource management training: results of a pragmatic trial. Journal of Evaluation in Clinical Practice: 2015, 21(1), 137-144

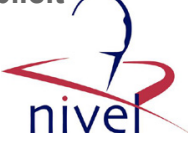

regarding the effect on the HI subset score and the EPOC overall score. In model 4, the intervention effect is 0.25 (95\% CI $0.06-0.43)$, meaning that the number of times explicit professional communication was observed during an interaction between to professionals in the intervention group was 0.25 tally higher than in the control group.

\section{[TABLE 6]}

Our study showed an increase in the use of explicit professional communication after CRM training in EDs observed by independent observers by means of the EPOC tool. After correction for days after training and safety culture, explicit communication increased with approximately one observable expression per four interaction moments.

We did not find an effect on the 'AoE' score, which included SA. We tried to measure existing SA in terms of 'names stressors (stress, fatigue, busyness)', 'takes action in line with stressors' and 'keeps patient overview'. Existing SA, however, is not necessarily overt SA, which is merely the expression of misperceptions and lack of knowledge. These were no part of the EPOC SA dimension. Other techniques, for instance self-ratings, may offer additional information about internal, implicit SA [20]. Future studies should consider various techniques thoroughly. We found increasing explicit communication over time in both groups, possibly because of the growing awareness of the importance of non-technical skills for patient safety during the follow-up period.

Because we expected the effect of the training to affect all the individuals in the department, we decided to observe many individuals for short periods of time instead of a few caregivers for a longer period. This could explain why observations do not seem to cluster within individuals. Possibly, the amount of explicit communication is more task dependent than dependent on individual characteristics.

One could argue whether an increase in communication is an indicator of increased safety. In the ED, we believe a small increase in explicit communication could have a significant impact on patient safety as care is sequenced. One may also suggest that an increase in interactions themselves could be an effect of CRM training. However, we think our scheme of explicit communication has included this increase, as an increase in communication about clinical conditions is usually observable as an EPOC item, for example, 'asks for input'. Our focus on observed EPOC items per interaction will therefore be sufficient to draw inferences about the effectiveness of CRM training.

Several methodological limitations must be mentioned. First, we had to exclude data of 71 observations because of incomplete data. Comparison of the first two models of the complete sample (Table 4) with the corresponding models on complete cases (Table 6) revealed that intervention effects were smaller in models using all available data. Thus, the effects of the fully corrected models are overestimated by approximately $5 \%$. Second, observers discovered during the post-training measurement, which departments got the intervention as trainees sometimes mentioned words such as 'situation awareness'. As a result, observer bias has to be taken into account. However, the structural design of EPOC limits the influence of subjectivity of the observers [13]. Third, a possible Hawthorne effect may have taken place as people may act differently when they feel they are judged by observers. 
Verbeek-van Noord, I., Bruijne, M.C. de, Twisk, J.W.R., Dyck, C. van, Wagner, C. More explicit communication after classroom-based crew resource management training: results of a pragmatic trial. Journal of Evaluation in Clinical Practice: 2015, 21(1), 137-144

Participants, however, said they got used to be observed very quickly, not being aware of being observed. Fourth, our sample size of four departments is low, but the number of observations and the finding that observations do not cluster within individuals increased the power significantly. Fifth, selection bias could be present as the selection of the intervention group was made on the basis of readiness to pay for the intervention and scheduling time off for staff. EDs that skillfully succeed in not scheduling all personnel for 2 days might be more motivated to comply with the training content. However, we believe that another mechanism plays a role, i.e. complacency. Previous research revealed that non-implementation of safety measures is predicted by several indicators of a favourable safety culture [16]. In other words, high culture scores may reflect naivety regarding the need for safety improvement. In the present study, the departments in the control group indeed scored significantly higher on all but two dimensions of the HSOPSC. We tried to capture this possible bias by means of adjusting for several culture variables. Sixth, we are aware a didactic CRM training needs an implementation plan for sustainment in clinical practice [21,22]. Practical tools like SBAR [23] as well as structural changes in the organization, on-site coaching and management commitment [21,22] would possibly have given a larger effect.

Regarding generalizability, we think that our results will hold for other EDs in the Netherlands, as care characteristics are more or less the same in every ED. With correction for culture covariates, cultural influences on behaviour are minimalized. However, other barriers (i.e. habits, environmental obstacles or lack of perceived ability) [24] that we did not take into account in this study may influence behaviour as well.

Overall, we believe $25 \%$ more explicit communication is a clinically relevant difference, albeit slightly overestimated. Our results still indicate that classroombased CRM training is a promising tool to instill safety critical behaviour. The explicit oral communication we measured is intended to prevent communication errors. An increase in explicit communication may decrease communication errors and their related adverse events. However, more research is needed to assess whether the effects are sustained and to assess the effects on patient outcomes.

\section{ACKNOWLEDGEMENTS}

The authors would like to thank the departments for their contribution to the study, especially the health care workers for letting them being observed and filling in the questionnaires.

\section{REFERENCES}

1 Kohn, L. T., Corrigan, J. M. \& Donaldson, M. S. (1999) To Err is Human: Building a Safer Health System. Washington, DC: Institute of Medicine.

2 Committee on Quality of Healthcare in America (2001) Crossing the Quality Chasm: A New Health System for the 21st Century. Washington, DC: Institute of Medicine.

3 National Quality Forum (2003) Safe Practices for Better Healthcare: A Consensus Report. Washington, DC: National Quality Forum.

4 Pizzi, L., Golfarb, N. I. \& Nash, D. B. (2001) Crew Resource Management and its Application in Medicine. Rockville, MD: Agency for Healthcare Research and Quality. Report No.: 01E058.2001. 
Verbeek-van Noord, I., Bruijne, M.C. de, Twisk, J.W.R., Dyck, C. van, Wagner, C. More explicit communication after classroom-based crew resource management training: results of a pragmatic trial. Journal of Evaluation in Clinical Practice: 2015, 21(1), 137-144

5 Croskerry, P. \& Sinclair, D. (2001) Emergency medicine: a practice prone to error? Canadian Journal of Emergency Medical Care, 3 (4), 271-276.

6 Endsley, M. R. (1988) Design and evaluation for situation awareness enhancement. Proceedings of the Human Factors and Ergonomics Society Annual Meeting, 32, 97-101. doi:10.1177/154193128803200221.

7 Endsley, M. R. (2000) Theoretical underpinnings of situation awareness: a critical review. In Situation Awareness Analysis and Measurement (eds M. R. Endsley \& D. J. Garland), pp. 3-28. Mahwah, NJ: Lawrence Erlbaum Associate, Inc., Publishers.

8 Rabol, L. I., Ostergaard, D. \& Mogensen, T. (2010) Outcomes of classroom-based team training interventions for multiprofessional hospital staff. A systematic review. Quality and Safety Health Care, 19 (6), e27. doi: 10.1136/qshc.2009.037184.

9 Weaver, S. J., Rosen, M. A., DiazGranados, D., et al. (2010) Does teamwork improve performance in the operating room? A multilevel evaluation. Joint Commission Journal on Quality and Patient Safety, 36 (3), 133-142.

10 Morey, J. C., Simon, R., Jay, G. D., Wears, R. L., Salisbury, M., Dukes, K. A. \& Berns, S. D. (2002) Error reduction and performance improvement in the emergency department through formal teamwork training: evaluation results of the MedTeams project. BMC Health Services Research, 37 (6), 1553-1581.

11 Musson, D. M. \& Helmreich, R. L. (2004) Team training and resource management in healthcare: current issues and future directions. Harvard Health Policy Review, 5, 25-35.

12 Tamuz, M. \& Harrison, M. I. (2006) Improving patient safety in hospitals: Contributions of high-reliability theory and normal accident theory. BMC Health Services Research, 41 (4 Pt 2), 1654-1676.

13 Kemper, P. F., van Noord, I., De Bruijne, M. C., Knol, D. L., Wagner, C. \& van Dyck, C. (2013) Development and reliability of the explicit professional oral communication (EPOC) observation tool to quantify the use of non-technical skills in health care. BMJ Quality and Safety, 22 (7), 586-595. doi: 10.1136/bmjqs-2012-001451.

14 Smits, M., Christiaans-Dingelhoff, I., Wagner, C., Wal, G. \& Groenewegen, P. P. (2008) The psychometric properties of the 'Hospital Survey on Patient Safety Culture' in Dutch hospitals. BMC Health Services Research, 8, 230. doi: 10.1186/1472-6963-8-230.

15 Sorra, J. S. \& Nieva, V. F. (2004) Hospital Survey on Patient Safety Culture. Rockville, MD: Agency for Healthcare Research on Quality.

16 van Noord, I., De Bruijne, M. C. \& Twisk, J. W. (2010) The relationship between patient safety culture and the implementation of organizational patient safety defences at emergency departments. International Journal for Quality in Health Care, 22 (3), 162-169. doi: $10.1093 /$ intqhc/mzq013.

17 van Dyck, C. (2000) Putting Errors to Good Use: Error Management Culture in Organizations. Amsterdam: University of Amsterdam.

18 Van Dyck, C., Frese, M., Baer, M. \& Sonnentag, S. (2005) Organizational error management culture and its impact on performance: a two-study replication. Journal of Applied Psychology, 90 (6), 1228-1240.

19 Rybowiak, V., Garts, H., Frese, M. \& Batinic, B. (1999) Error orientation questionnaire (EOQ): reliability, validity and different language equivalent. Journal of Organizational Behaviour, 20, 527-547.

20 Jones, D. G. (2000) Subjective measures of situation awareness. In Situation Awareness Analyses and Measurement (eds M. R. Endsley \& D. J. Garland), pp. 113-128. New York, NY: CRC Press.

21 Salas, E., Almeida, S. A., Salisbury, M., King, H., Lazzara, E. H., Wilson, K. A., Almeida, P. A. \& McQuilllan, R. (2009) What are the critical success factors for team training in health care? The Joint Commission Journal on Quality and Patient Safety, 35 (8), 398-405.

22 Morey, J. C. \& Salisbury, M. (2002) Introducing teamwork training into healthcare organizations: implementation issues and solutions. Proceedings of the Human Factor and Ergonomics Society Annual Meeting, 46 (25), 2069-2073. doi:10.1177/154193120204602516.

23 De Meester, K., Verspuy, M., Monsieurs, K. G. \& Van Bogaert, P. (2013) SBAR improves nurse-physician communication and reduces unexpected death; a pre and post intervention study. Resuscitation, 84 (9), 1192-1196. doi:

10.1016/j.resuscitation.2013.03.016. 
Verbeek-van Noord, I., Bruijne, M.C. de, Twisk, J.W.R., Dyck, C. van, Wagner, C. More explicit communication after classroom-based crew resource management training: results of a pragmatic trial. Journal of Evaluation in Clinical Practice: 2015, 21(1), 137-144

24 Perkins, M. B., Jensen, P. S., Jaccard, J., Gollwitzer, P., Oettingen, G., Pappadopulos, E. \& Hoagwood, K. E. (2007) Applying theory-driven approaches to understanding and modifying clinicians' behavior: what do we know? Psychiatric Services, 58 (3), 342-348.

\section{TABLES}

Table 1 Description of the crew resource management training

\begin{tabular}{|c|c|c|}
\hline Themes & Objectives & Educational methods \\
\hline Introduction & $\begin{array}{l}\text { Trainers introduced to trainees; trainees introduced to trainers; } \\
\text { trainers explain the goal of the course, the educational methods; } \\
\text { guaranteeing confidentiality. }\end{array}$ & Oral communication; exercise \\
\hline Role within a team & $\begin{array}{l}\text { To investigate what someone's role within a team is and how this } \\
\text { team relates to the organization. }\end{array}$ & $\begin{array}{l}\text { Plenary session; group discussion; assignment in } \\
\text { subgroups; cockpit audiotape air crash Tiger } 66\end{array}$ \\
\hline Human factors & $\begin{array}{l}\text { To explain how human errors could happen when individuals } \\
\text { interact with others and their environment. }\end{array}$ & Plenary session; group discussion \\
\hline $\begin{array}{l}\text { From blame to just } \\
\text { culture }\end{array}$ & $\begin{array}{l}\text { To explain that it is human to make mistakes and that it is only in } \\
\text { cases of serious negligence that someone can fully be } \\
\text { responsible for his own deeds. }\end{array}$ & Plenary session; group discussion \\
\hline Threats and errors & $\begin{array}{l}\text { To make clear that there are latent threats (e.g. organizational } \\
\text { culture, insufficient resources) and visible threats (e.g. } \\
\text { environmental, individual and team factors) to safety. }\end{array}$ & $\begin{array}{l}\text { Plenary session; group discussion; assignment in } \\
\text { subgroups with the HFACS* checklist }\end{array}$ \\
\hline $\begin{array}{l}\text { Situation awareness } \\
\text { and decision } \\
\text { making }\end{array}$ & $\begin{array}{l}\text { To explain what SA is and how it could be established, how a high } \\
\text { team SA could lead to better decisions and how a loss of SA } \\
\text { could occur. }\end{array}$ & $\begin{array}{l}\text { Plenary session; group discussion; accident with } \\
\text { Southwest Airlines }\end{array}$ \\
\hline $\begin{array}{l}\text { Communication and } \\
\text { teamwork }\end{array}$ & $\begin{array}{l}\text { To clarify how communication/miscommunication works and how } \\
\text { effectively teams communicate. }\end{array}$ & $\begin{array}{l}\text { Plenary session; group discussion; group exercise; } \\
\text { group assignment (team-building activity Zin } \\
\text { Obelisk) }\end{array}$ \\
\hline Important tips & $\begin{array}{l}\text { To translate that what was learned on the first day into practical } \\
\text { tips and tools for everyday practice. }\end{array}$ & $\begin{array}{l}\text { Assignment: make action points according to what } \\
\text { has been discussed }\end{array}$ \\
\hline $\begin{array}{l}\text { Situational } \\
\text { leadership }\end{array}$ & $\begin{array}{l}\text { To explain that team effectiveness depends on the quality of } \\
\text { leadership multiplied by the acceptance by the team. To explain } \\
\text { that every situation, team or individual needs different styles of }\end{array}$ & $\begin{array}{l}\text { Plenary session; group discussion; assignment: } \\
\text { describe team around patient at the ED; test: } \\
\text { personality styles }\end{array}$ \\
\hline
\end{tabular}

Groupthink leadership. To outline the features of an effective leader.

To describe what groupthink is, how it could disturb team effectiveness, and how one could minimize its influence

Stress management To define what stress is, how one can recognize it, what its effect is, how it is caused and how it could be solved.

Individual influences within a team

To make clear that all individual behaviour is influenced by internal processes and conditions and that some characteristics of the individual are known by the individual itself and/or the team or by neither of them (blind spot). To name the five hazardous attitudes.

Giving feedback To introduce the concept of feedback and self-reflection by explaining how quality in characters (e.g. self-confidence) can become risks (e.g. arrogance)

Plenary session; group discussion; video NASA

Plenary session; group discussion; assignment: Holmes-Rahe stress scale

Plenary session; group discussion; assignment: describe behaviour during a videotaped political debate

Plenary session; group discussion; exercise 
Verbeek-van Noord, I., Bruijne, M.C. de, Twisk, J.W.R., Dyck, C. van, Wagner, C. More explicit communication after classroom-based crew resource management training: results of a pragmatic trial. Journal of Evaluation in Clinical Practice: 2015, 21(1), 137-144

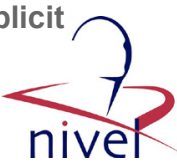

\begin{tabular}{|c|c|c|c|c|c|c|}
\hline & \multicolumn{2}{|c|}{ Intervention group } & \multicolumn{2}{|c|}{ Control group } & \multicolumn{2}{|l|}{ Total } \\
\hline & $\begin{array}{l}n \\
\text { Sub. } \\
\left(\%^{*}\right)\end{array}$ & $\begin{array}{l}n \\
\text { Obs. } \\
\left(\%^{+}\right)\end{array}$ & $\begin{array}{l}n \\
\text { Sub. } \\
\left(\%^{*}\right)\end{array}$ & $\begin{array}{l}n \\
\text { Obs. } \\
\left(\%^{+}\right)\end{array}$ & $\begin{array}{l}n \\
\text { Sub. } \\
\left(\%{ }^{*}\right)\end{array}$ & $\begin{array}{l}n \\
\text { Obs. } \\
\left(\%^{\dagger}\right)\end{array}$ \\
\hline Eligible for the study & $\begin{array}{r}79 \\
(100)\end{array}$ & & $\begin{array}{r}70 \\
(100)\end{array}$ & & $\begin{array}{r}149 \\
(100)\end{array}$ & \\
\hline Observations at baseline & $\begin{array}{r}59 \\
(75)\end{array}$ & 100 & $\begin{array}{r}52 \\
(74)\end{array}$ & 82 & $\begin{array}{l}111 \\
(74)\end{array}$ & 182 \\
\hline Observations after intervention & $\begin{array}{r}48 \\
(61)\end{array}$ & $\begin{array}{r}94 \\
(100)\end{array}$ & $\begin{array}{r}38 \\
(54)\end{array}$ & $\begin{array}{r}84 \\
(100)\end{array}$ & $\begin{array}{r}86 \\
(58)\end{array}$ & $\begin{array}{r}178^{ \pm} \\
(100)\end{array}$ \\
\hline Complete data on culture & $\begin{array}{r}34 \\
(43)\end{array}$ & $\begin{array}{r}65 \\
(69)\end{array}$ & $\begin{array}{r}31 \\
(44)\end{array}$ & $\begin{array}{r}71 \\
(85)\end{array}$ & $\begin{array}{r}65 \\
(44)\end{array}$ & $\begin{array}{r}136^{5} \\
(76)\end{array}$ \\
\hline
\end{tabular}

Table 2 Numbers of observed subjects (sub.) and observations (obs.)

*Percentages in this second, third and fourth row of this column represent the \% of persons compared with the number of persons that were eligible for the study (first row = denominator).

${ }^{\dagger}$ Percentage in the fourth row of this column represent the $\%$ of observations compared with the third row (= denominator) of this column.

'Number of observations used in models unadjusted for safety culture dimensions (Table 4).

${ }^{5}$ Number of observations used in models adjusted for safety culture dimensions (Table 6).

Table 3 Description of directly observed explicit professional oral communication scores: number of tallies on each EPOC category and dimension* and their corresponding median (25th-75th percentile) score

\begin{tabular}{|c|c|c|c|c|c|c|c|c|c|}
\hline & & \multicolumn{4}{|c|}{ Pre-training measurement } & \multicolumn{4}{|c|}{ Post-training measurement } \\
\hline & & \multicolumn{2}{|c|}{ Intervention } & \multicolumn{2}{|c|}{ Control } & \multicolumn{2}{|c|}{ Intervention } & \multicolumn{2}{|c|}{ Control } \\
\hline \multicolumn{2}{|l|}{$n$ interactions } & \multicolumn{2}{|l|}{857} & \multicolumn{2}{|l|}{618} & \multicolumn{2}{|l|}{966} & \multicolumn{2}{|l|}{735} \\
\hline \multicolumn{2}{|l|}{$n$ observations } & \multicolumn{2}{|l|}{100} & \multicolumn{2}{|l|}{79} & \multicolumn{2}{|l|}{107} & \multicolumn{2}{|l|}{92} \\
\hline \multicolumn{2}{|c|}{ Mean (SD) interactions per observation } & \multicolumn{2}{|c|}{$4.5(5.8)^{\dagger}$} & \multicolumn{2}{|c|}{$2.9(4.8)^{\dagger}$} & \multicolumn{2}{|c|}{$5.1(5.7)^{\dagger}$} & \multicolumn{2}{|c|}{$3.5(4.7)^{\dagger}$} \\
\hline EPOC category & EPOC dimension & $n$ & Median & $n$ & Median & $n$ & Median & $n$ & Median \\
\hline Self & Assertiveness & 7 & $0.0(0.0-0.0)$ & 4 & $0.0(0.0-0.0)$ & 1 & $0.0(0.0-0.0)$ & 3 & $0.0(0.0-0.0)$ \\
\hline \multicolumn{2}{|c|}{ Human interaction } & 772 & $1.0(0.6-1.3)$ & 528 & $0.9(0.6-1.3)$ & 1058 & $1.1(1.0-1.4)$ & 702 & $1.0(0.7-1.2)$ \\
\hline \multicolumn{2}{|c|}{ Working with others } & 401 & $0.5(0.3-0.8)$ & 265 & $0.4(0.3-0.7)$ & 612 & $0.7(0.5-0.9)$ & 409 & $0.6(0.4-0.8)$ \\
\hline \multicolumn{2}{|c|}{ Task-oriented leadership } & 124 & $0.1(0.0-0.2)$ & 87 & $0.1(0.0-0.3)$ & 74 & $0.0(0.0-0.1)$ & 50 & $0.0(0.0-0.1)$ \\
\hline \multicolumn{2}{|c|}{ People-oriented leadership } & 247 & $0.3(0.2-0.5)$ & 176 & $0.3(0.1-0.5)$ & 372 & $0.4(0.3-0.5)$ & 243 & $0.3(0.2-0.5)$ \\
\hline \multicolumn{2}{|c|}{ Anticipation on environment } & 107 & $0.0(0.0-0.2)$ & 75 & $0.1(0.0-0.2)$ & 80 & $0.0(0.0-0.1)$ & 43 & $0.0(0.0-0.1)$ \\
\hline \multicolumn{2}{|c|}{ Situation awareness } & 41 & $0.0(0.0-0.1)$ & 43 & $0.0(0.0-0.1)$ & 36 & $0.0(0.0-0.1)$ & 22 & $0.0(0.0-0.0)$ \\
\hline \multicolumn{2}{|c|}{ Planning and anticipating } & 66 & $0.0(0.0-0.1)$ & 32 & $0.0(0.0-0.1)$ & 44 & $0.0(0.0-0.0)$ & 21 & $0.0(0.0-0.0)$ \\
\hline \multicolumn{2}{|c|}{ Overall EPOC score } & 886 & $1.1(0.8-1.5)$ & 607 & $1.0(0.8-1.4)$ & 1139 & $1.2(1.0-1.4)$ & 748 & $1.0(0.8-1.3)$ \\
\hline
\end{tabular}

*Each EPOC category could be further subdivided into EPOC dimensions (only for the Self category, only one dimension is defined).

${ }^{\dagger}$ Significant difference between intervention and control group. 
Verbeek-van Noord, I., Bruijne, M.C. de, Twisk, J.W.R., Dyck, C. van, Wagner, C. More explicit communication after classroom-based crew resource management training: results of a pragmatic trial. Journal of Evaluation in Clinical Practice: 2015, 21(1), 137-144

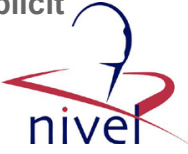

Table 4 The effect of the CRM training on human interaction, the overall EPOC score and anticipation on environment $(n=178)^{*}$

\begin{tabular}{llllll}
\hline & \multicolumn{1}{l}{ Model 1 } & & Model 2 & \\
\cline { 2 - 3 } Outcome & $\beta(95 \% \mathrm{Cl})$ & & & $\beta(95 \% \mathrm{Cl})$ & $P$ \\
\hline EPOC overall & $0.14(0.02-0.26)$ & $<0.025$ & & $0.19(0.07-0.31)$ & $<0.005$ \\
Human interaction & $0.13(0.00-0.25)$ & $<0.05$ & & $0.18(0.05-0.30)$ & $<0.005$ \\
\hline & OR $(95 \% \mathrm{Cl})$ & $P$ & & OR $(95 \% \mathrm{Cl})$ & $P$ \\
\hline Anticipation on & $1.50(.81-2.77)$ & $<0.25$ & & $1.34(0.71-2.52)$ & $>0.25$ \\
environment & & & & \\
\hline
\end{tabular}

*Models are derived from linear for logistic mixed models analyses.

Model 1 = crude model: model adjusted for baseline measurement of outcome variable.

Model 2 = model 1 plus correction for days between training and observation [mean 112 days after training (range 71 to 148 days)].

\begin{tabular}{lll}
\hline Patient safety culture dimension & $\begin{array}{l}\text { Intervention } \\
\text { group }(n=39)\end{array}$ & $\begin{array}{l}\text { Control group } \\
(n=27)\end{array}$ \\
\hline Smooth transitions & $3.1(0.8)$ & $3.3(0.7)$ \\
Frequency of event reporting* & $2.8(0.9)$ & $3.2(0.8)$ \\
Non-punitive response to error* & $3.4(0.5)$ & $3.7(0.6)$ \\
Feedback about and learning from error* $^{*}$ & $3.1(0.6)$ & $3.6(0.6)$ \\
Hospital management support* & $2.8(0.6)$ & $3.3(0.6)$ \\
Adequate staffing* & $3.5(0.4)$ & $3.1(0.6)$ \\
\hline Error management culture dimension & & $3.7(0.5)$ \\
\hline Mastery* & $3.6(0.3)$ & $3.6(0.6)$ \\
Social orientation* & $3.4(0.4)$ & $2.5(0.6)$ \\
Awareness & $2.5(0.4)$ & $2.5(0.4)$ \\
Aversion* & $2.7(0.4)$ & \\
\hline
\end{tabular}

Table 5 Patient safety and error management culture at baseline [mean (SD)] in observed subjects based on a 5-point Likert scale

*Indicates a statistically significant difference between intervention and control group $(P \leq 0.05)$ based on a $t$-test for independent samples. 
Verbeek-van Noord, I., Bruijne, M.C. de, Twisk, J.W.R., Dyck, C. van, Wagner, C. More explicit communication after classroom-based crew resource management training: results of a pragmatic trial. Journal of Evaluation in Clinical Practice: 2015, 21(1), 137-144

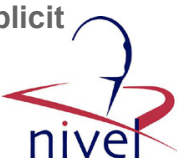

Table 6 The effect of the CRM training on the overall EPOC score, human interaction and anticipation on environment score ( $n=136)$, adjusted for 10 culture covariates*

\begin{tabular}{|c|c|c|c|c|c|c|c|c|}
\hline \multirow[b]{2}{*}{ Outcome } & \multicolumn{2}{|l|}{ Model 1} & \multicolumn{2}{|l|}{ Model 2} & \multicolumn{2}{|l|}{ Model 3} & \multicolumn{2}{|l|}{ Model 4} \\
\hline & $\beta(95 \% \mathrm{Cl})$ & $P$ & $\beta(95 \% \mathrm{Cl})$ & $P$ & $\beta(95 \% \mathrm{Cl})$ & $P$ & $\beta(95 \% \mathrm{Cl})$ & $P$ \\
\hline EPOC overall score & $0.19(0.05-0.32)$ & $<0.01$ & $0.23(0.10-0.37)$ & 0.001 & $0.25(0.07-0.43)$ & $<0.01$ & $0.25(0.06-0.43)$ & $<0.01$ \\
\hline Human interaction & $0.17(0.03-0.32)$ & $<0.025$ & $0.22(0.07-0.37)$ & $<0.005$ & $0.25(0.07-0.44)$ & $<0.01$ & $0.27(0.08-0.45)$ & $<0.01$ \\
\hline Outcome & $\mathrm{OR}(95 \% \mathrm{Cl})$ & $P$ & OR $(95 \% \mathrm{Cl})$ & $P$ & OR $(95 \% \mathrm{Cl})$ & $P$ & OR $(95 \% \mathrm{Cl})$ & $P$ \\
\hline $\begin{array}{l}\text { Anticipation on } \\
\text { environment }\end{array}$ & $1.53(0.75-3.11)$ & $<0.25$ & $1.38(0.65-2.96)$ & $>0.25$ & $0.99(0.35-2.79)$ & $>0.25$ & $0.71(0.23-2.15)$ & $>0.25$ \\
\hline
\end{tabular}

*Models are derived from linear for logistic mixed models analyses.

Model 1 = crude model: model adjusted for baseline measurement of outcome variable.

Model 2 = model 1 plus correction for days between training and observation [mean 111 days after training (range 71 to 148 days)].

Model 3 = model 2 plus correction for patient safety culture dimensions: smooth transitions, frequency of error reporting, non-punitive response to error, feedback about and learning from errors, hospital management support for patient safety and staffing.

Model 4 = model 3 plus correction for error management culture dimensions: mastery, social orientation, awareness and aversion. 